\title{
Criminologie
}

\section{Institut Philippe Pinel et la recherche évaluative}

\section{Sheilagh Hodgins}

Volume 15, numéro 2, 1982

Criminels et psychiatrie

URI : https://id.erudit.org/iderudit/017162ar

DOI : https://doi.org/10.7202/017162ar

Aller au sommaire du numéro

Éditeur(s)

Les Presses de l'Université de Montréal

ISSN

0316-0041 (imprimé)

1492-1367 (numérique)

Découvrir la revue

Citer cet article

Hodgins, S. (1982). Institut Philippe Pinel et la recherche évaluative.

Criminologie, 15(2), 93-104. https://doi.org/10.7202/017162ar d'utilisation que vous pouvez consulter en ligne.

https://apropos.erudit.org/fr/usagers/politique-dutilisation/ 
L'Institut Philippe Pinel de Montréal est un centre hospitalier psychiatrique qui assure des contrats de service pour l'évaluation et le traitement des détenus en provenance des diverses institutions pénales du Québec. Une unité ayant une capacité de 21 patients, l'Institut compte quinze unités de service dont deux sont directement affectées à des évaluations psychiatriques pour des fins de cour.

L'Institut Philippe Pinel regroupe différents types de clientèle en traitement. L'une de ces clientèles est constituée d'individus emprisonnés en vertu d'un mandat du lieutenant-gouverneur. Il s'agit d'individus accusés d'un crime mais jugés inaptes à subir leur procès ou encore acquittés pour raison d'aliénation mentale ${ }^{1}$. Officiellement incarcérés " au bon plaisir du lieutenant-gouverneur ", de tels patients relèvent de la responsabilité de la Commission d'examen qui revise leurs cas au moins une fois par année et qui, après consultation auprès du personnel traitant, prend les décisions qui s'imposent concernant leurs libération, privilèges, et autres.

À l'Institut, on retrouve également un autre type de clientèle, à savoir la clientèle des détenus des institutions pénitencières fédérales ou provinciales qui ont développé des problèmes psychologiques graves pendant leur incarcération et qui ont été temporairement transférés pour y être traités. Après amélioration de leur condition de santé, ils retournent à leur prison d'origine.

Une troisième catégorie de détenus comprend la clientèle des institutions fédérales ou provinciales inscrites à des programmes de réhabilitation. Il s'agit d'individus qui ont été sélectionnés à partir de leur potentiel élevé de réussite et qui, sur une base volontaire, ont manifesté le désir de participer à l'un de ces programmes. Finalement, ont peut retrouver à l'Institut des détenus qui sont en cure

* Toutes les observations, les opinions, les commentaires émis dans le texte n'appartiennent qu'à l'auteur et n'engagent d'aucune façon l'Institut Philippe Pinel. Néanmoins, l'IPPM soutient la démarche de recherche de l'auteur.

* Sheilagh Hodgins, psychologues, à l'École du service social, Université de Montréal.

1. Même si la cour a officiellement "acquitté » ces individus, ils sont quand même traités pour des fins cliniques, comme s'ils avaient commis le crime dont ils sont accusés. 
fermée et qui présenteraient un certain danger dans un hôpital psychiatrique, mais leur nombre est très limité.

En général, le personnel de l'Institut se perçoit comme un personnel clinique qui travaille dans un hôpital psychiatrique. Ả l'intérieur de l'institution, le langage, l'ambiance et les activités ressemblent beaucoup à ceux des autres institutions psychiatriques. Chaque unité de traitement compte une équipe multidisciplinaire qui inclut un psychiatre, un psychologue, un criminologue, des infirmières, des sociothérapeutes, des éducateurs et des agents communautaires. En outre, chaque unité présente un programme de traitement qui lui est propre et qui doit desservir une clientèle plus ou moins homogène.

De tout cela, il ressort que l'Institut est le seul de ce type au Québec. Il dessert une population peu nombreuse et il offre la sécurité d'une prison et les services d'un hôpital psychiatrique. C'est donc dire que chaque patient coûte très cher et c'est la raison pour laquelle il importe d'exiger une évaluation qui démontrerait l'efficacité de ses interventions. Telle ne semble pas être pourtant la logique dans le domaine des programmes de réhabilitation auprès des criminels (Seechrest, White \& Brown, 1979), ni dans celui du traitement des difficultés psychologiques (Rachman \& Wilson, 1980)

Conscient de ses responsabilités, on ne peut que féliciter l'IPPM pour la décision qu'il a prise d'améliorer l'efficacité et l'économie de ses services en encourageant un projet comme le nôtre.

\section{La stratégie adoptée}

Afin d'évaluer les traitements offerts dans l'institution, on peut procéder de trois façons. On peut d'abord demander une évaluation aux experts en recherche évaluative, ou encore, on peut le demander aux chercheurs attachés au Service de Recherche de l'IPPM. De telles alternatives n'ont pas, cependant, grande chance de succès, car elles risquent d'être continuellement sabotées par le personnel clinique qui lui n'a pas tellement de pouvoir concret dans ce processus de recherche. Selon cette perspective, au lieu d'y assumer un rôle actif, il joue surtout un rôle passif car il est l'objet de la recherche. La nature même du processus de recherche impliquée dans ces alternatives ne convient pas à nos vrais besoins car les informations ainsi obtenues, sur le plan quantitatif ou qualitatif, seraient trop statiques pour améliorer l'efficacité des interventions. Etant donné les limites des connaissances scientifiques et évaluati- 
ves en ce domaine, il est préférable de partir du travail clinique qui est en même temps un effort pour changer le patient et une tentative pour développer un corpus de connaissances.

Le raisonnement qui nous a amené à arriver à cette conclusion est présenté ailleurs (Hodgins, 1982), cependant, il est important de le reprendre brièvement ici. Même si on ne peut le justifier valablement, il n'en reste pas moins que le manque de connaissances dans le domaine de la criminologie clinique est effarant. En général, les évaluations de thérapie auprès des adultes qui veulent changer un état de psychose, de dépression ou même un ou plusieurs comportements, attitudes ou sentiments, sont un échec (Berbin \& Garfield, 1978). Le fait de ne pas avoir de traitements jugés efficaces auprès des criminels-malades mentaux est donc tout à fait logique. On explique également cette lacune par le fait que la science comprend mal le développement des comportements criminels chez un individu ainsi que le développement de ses difficultés psychologiques. Jusqu'à présent, le lien entre un comportement criminel et une maladie mentale nous laisse dans l'impossibilité de prédire qui pourrait tomber malade et qui pourrait devenir dangereux. C'est à partir de ce manque de connaissances flagrant que les cliniciens de l'IPPM doivent travailler.

Compte tenu de cette situation, nous disons que la recherche évaluative, - et c'est la troisième façon de voir et qui est aussi la nôtre, - doit devenir partie intégrante du travail clinique de façon à ce que les cliniciens arrivent à réaliser que les traitements qu'ils proposent ne sont que des hypothèses à confirmer ou à infirmer. Un tel esprit empirique reconnaît à la base le manque de connaissances et favorise l'implantation d'une stratégie d'interventions efficaces.

Dans la perspective où la recherche et le travail clinique marcheraient la main dans la main dans un esprit empirique, tout programme d'intervention mis de l'avant aurait une base théorique appuyée sur des données. Dès sa mise en application un tel programme serait constamment enrichi suite à l'accumulation de nouvelles données objectivement quantifiables, la dite accumulation se poursuivant au-delà de l'application du traitement. Ces données nous renseigneraient non seulement sur les possibilités de cette intervention à réduire le comportement criminel et les difficultés psychologiques chez le sujet traité, mais aussi sur l'interaction entre les différents éléments du programme et les différents types de sujet.

Il y a une autre raison qui nous fait opter en faveur d'une stra- 
tégie d'évaluation continuelle du traitement du client. Notre argument est fondé sur le bien-être de l'individu comme tel. Les différentes recherches qui évaluent les programmes d'interventions auprès des multiples catégories de patients concluent que ceux sous traitement n'ont pas véritablement amélioré leur comportement. Au mieux, il est resté inchangé; au pire, il s'est détérioré. Et jusqu'à présent, cette conclusion demeure vraie quelles que soient les formes de traitements proposées (Lambert, Bergin \& Collins, 1977). On ne possède pas d'informations nécessaires pour identifier le type de patient par rapport au sens de sa réaction (Bergin \& Garfield, 1978). Éthiquement, on se voit donc contraint d'observer l'évolution du comportement des patients pour au moins faire cesser le traitement chez ceux dont le comportement se détériore en conséquence.

\section{Les difficultés rencontrées}

Cette stratégie de cueillette continue d'informations sur l'évolution du client est la seule qui pourrait éventuellement nous fournir des hypothèses qui par la suite seraient vérifiées par le biais d'expériences plus classiques et aboutiraient à des programmes de traitement plus efficaces. Cependant, l'application d'une telle stratégie implique des difficultés de différents ordres.

L'IPPM est un hôpital qui doit fournir des services vingt-quatre heures par jour avec un budget très déterminé. En outre, il y a des exigences d'ordre médical et légal, souvent non prévisibles et sur lesquelles l'IPPM ne peut exercer de contrôle. Le personnel traitant est occupé et doit faire face à des situations qui demandent continuellement des réponses immédiates. De telles activités cliniques reposent sur une orientation théorique et une formation préalable. Pour orienter son travail, il faut que le personnel croit à ce fondement théorique et aux types d'intervention qui en découlent. C'est tout le contraire de l'attitude empirique nécessaire à la recherche.

Comme Quinsey (1981) l'a lui-même fait remarquer, les programmes de traitement ne sont pas mis sur pied selon une étude de littérature scientifique mais plutôt selon le goût et la formation du personnel engagé pour les gérer. En général, les cliniciens sont mal à l'aise avec tout ce qui s'appelle la recherche. Manquant de formation en ce domaine, ils la comprennnent mal; ils n'en voient pas l'utilité pour leur travail et ils en ont peur car ils craignent d'être évalués.

À tout cela, il faut ajouter que l'IPPM, comme les autres insti- 
tutions de ce genre est un milieu qui exerce un effet décourageant autant sur le personnel que sur les clients. Le personnel clinique a l'impression que c'est difficile de faire des progrès avec une telle clientèle. Les changements positifs, s'ils surviennent, arrivent lentement et petit à petit. Un autre élément vient compliquer la situation et ajouter un autre effet de démoralisation puisque malgré les efforts intensifs du personnel pour aider un patient à améliorer son comportement, ce dernier doit, de toute façon retourner par la suite au pénitencier pour terminer sa sentence. Alors s'il a été condamné à vingt-cinq ans, ce n'est pas demain la veille...

Une autre difficulté propre à une institution comme l'IPPM, c'est la formation du personnel. Personne n'a été préparée à travailler auprès d'une telle population. Chacun aurait reçu une formation "psychiatrique " ou une formation " criminologique" donc il faut développer les habiletés cliniques du personnel en cours d'emploi.

\section{Le projet d'évaluation}

Dans ce contexte la stratégie de recherche évaluative que l'on propose est à la fois un processus de recherche et de formation. Les cliniciens constituent une source d'informations extraordinaire. Et pour utiliser leurs connaissances, il faut d'abord les systématiser et les examiner à la loupe afin de distinguer celles qui ont prises sur une certaine réalité de vie de celles qui n'en ont pas. En étudiant de la sorte les informations de ceux et celles qui vivent le plus près possible de cette clientèle, à l'aide d'une méthodologie rigoureuse, on pourra départager le côté mystique de la clinique de son côté scientifique. C'est là une stratégie qui a une chance de déboucher sur un corpus de connaissances et d'interventions efficaces.

L'un des objectifs majeurs de tous les programmes de traitement de l'IPPM consistte, à n'en point douter, d'aider les patients à changer leurs comportements de façon à ce qu'ils ne commettent plus d'activités criminelles. Pour les gens atteints d'une maladie mentale ou d'une certaine arriération, l'objectif de traitement visé à éliminer les symptômes et à rendre les individus suffisamment autonomes pour pouvoir au moins habiter en dehors d'une institution.

D'après l'orientation théorique de l'équipe et la clientèle donnée, le programme de traitement de chaque unité cherche à atteindre cet objectif principal d'une façon plus ou moins claire et distincte. À partir de l'orientation théorique de l'équipe et des besoins in- 
dividuels du client, le programme de traitement poursuit des sousobjectifs qui nécessairement doivent être atteints par le patient afin de rejoindre l'objectif principal.

Prenons l'exemple d'un client habité par des hallucinations où une voix lui dit que s'il ne tue pas son frère qui est l'instrument du diable, Dieu se verra dans l'obligation de le torturer. Pour empêcher que le client récidive, - ceci est l'objectif principal, - l'équipe devra travailler à l'élimination des hallucinations de ce client - et c'est ce qu'on appelle un sous-objectif. Étant donné l'orientation de l'équipe, la méthode privilégiée pour atteindre ce sous-objectif pourra être l'utilisation d'un tranquilisant majeur accompagné d'un support affectif de groupe et d'un peu de thérapie du milieu. Même si les sous-objectifs sont déterminés à l'intérieur du cadre théorique du programme, on peut donc dire qu'ils sont individualisés pour répondre aux besoins de chaque cas. volets :

C'est pourquoi le projet d'évaluation que l'on réalise a trois

1. développer les instruments à chaque unité pour observer l'évolution continue des patients;

2. valider ces instruments et évaluer le produit du traitement en mesurant la récidive criminelle et les rechutes psychiatriques des ex-patients;

3. mesurer l'agression chez les patients et la réponse à de tels actes de la part du personnel traitant.

\section{Les instruments d'évolution}

On a tenté de bâtir des instruments qui mesurent l'évolution du patient afin de fournir aux différentes équipes de soin les informations dont elles avaient besoin pour modifier leurs propres interventions et rejoindre ainsi les sous-objectifs personnalisés de chaque patient. Il s'agissait de produire un instrument suffisamment sensible pour détecter les variations continuelles du comportement des patients, peu importe l'orientation de ces comportements. L'instrument ainsi produit peut être utilisé par un ou plusieurs membres de l'équipe. Il doit donc être fiable et de par sa nature même il vient limiter les biais d'intervention introduits par les cliniciens.

Étant donné des différences importantes dans les clientèles et dans les unités, on a construit plusieurs instruments. On a d'abord divisé les unités de soins en quatre catégories :

1. les unités d'admission; 
2. les unités de psychotiques dans lesquelles on peut retrouver certains cas d'arriération;

3 . les unités de soins prolongés;

4. les unités de criminels étiquettés " caractériels ».

Même si cette division est loin d'être parfaite, on peut dire qu'en général elle respecte la réalité que l'on a à traiter. Chaque unité a donc nommé un délégué qui travaille en groupe avec les délégués des autres unités, un cadre de service hospitalier et un consultant de la recherche évaluative.

Comme dans les unités d'admission et les unités de psychotiques les équipes travaillent sous pression constante pour faire disparaître les symptômes de la psychose; elles s'attendent à voir apparaître assez rapidement des changements dans les comportements de leurs patients. On a donc constaté le besoin commun de ces deux unités d'avoir un instrument qui mesurerait les symptômes des différentes formes de psychoses à partir desquels on pourrait identifier chaque semaine des changements ou un manque de changements.

Travaillant à partir des échelles qui existaient déjà et des descriptions de symptômes de ces désordres, on a construit une liste de quarante comportements observables. Cette liste a par la suite donné naissance à dix catégories descriptives de l'état clinique du patient. À l'intérieur de chacune de ces catégories, on a inséré une échelle graduée allant de "pas de difficulté " dans ce domaine jusqu'à " une difficulté majeure ».

On a revisé à plusieurs reprises la liste des comportements afin de s'assurer que les mots avaient la même signification pour tous les membres du personnel. On a fait de même au niveau de l'identification des catégories et de leur signification en ce qui a trait à leur contenu clinique pour les membres des équipes. On a réalisé de petites expériences de vérification de la fiabilité et de la validité de l'instrument.

Jusqu'à présent, on en a vérifié la validité de deux façons : premièrement, on a comparé les résultats de l'échelle de catégorisation avec les jugements cliniques des équipes; deuxièmement on a vérifié si l'instrument était sensible aux variations de comportements des patients observés par les équipes traitantes. On se retrouve donc actuellement avec un nouvel instrument qui semble avoir une certaine pertinence clinique et l'on s'empresse maintenant de procéder à la vérification de la fiabilité du dit instrument dans un projet beaucoup plus large et à plus long terme. 
À ce stade-ci de notre opération quelques remarques simposent. Il nous faut d'abord dire qu'à l'intérieur des unités de psychotiques, c'est dans la première phase du traitement de patients que notre instrument est pertinent. Il nous faut travailler une autre façon de mesurer leur évolution car dans les autres phases du traitement, le personnel de soins doit poursuivre d'autres sous-objectifs.

En travaillant avec les délégués des unités de soins prolongés on a très tôt reconnu que les changements observés chez leurs patients étaient minces et produits à un rythme tellement lent que l'on risquait de décourager tout le personnel traitant si on ne modifiait pas notre façon de mesurer leurs comportements. On s'est donc mis à construire un instrument qui pourra identifier de petits changements chez cette population et que l'on pourra appliquer sur des intervalles de temps plus longs afin d'augmenter la possibilité d'observer des changements de comportements positifs. Il convient de continuer dans ce domaine.

Du côté des unités de criminels ou de caractériels comme on les appelle dans le milieu, il restait une tâche immense à réaliser. On se devait de construire un instrument qui mesurerait l'évolution d'un homme de type criminel par rapport à celle d'un homme de type non criminel. Devant ce problème de taille, on a adopté une stratégie empirique qui tenait compte des connaissances cliniques du personnel au sujet de leur propre clientèle. À priori, on a tenté de construire une liste qui définissait les capacités de fonctionnement interpersonnel d'un homme dans laquelle on pouvait distinguer un criminel d'un non-criminel. Par la suite, on a demandé aux professionnels de la clinique de porter un jugement de valeur quant au degré de présence ou d'absence de ces différents traits de comportement chez tout être humain. Ainsi, l'on a constitué une grille que l'on a appliquée par la suite auprès de trois groupes d'individus : les caractériels de l'IPPM; un groupe de prisonniers dans un pénitencier; un groupe d'hommes à peu près du même âge et vivant à l'extérieur de toute institution.

Si l'on parvient à valider grosso-modo notre instrument, nous passerons par la suite à l'examen item par item de chaque capacité de fonctionnement interpersonnel de façon à conserver les items qui discriminent vraiment nos deux populations et à rejeter les autres. Nous ferons donc une autre expérimentation de façon à valider notre instrument item par item.

Toutes ces activités ne sont qu'une première étape dans l'éta- 
blissement des instruments qui visent à mesurer de façon continue des changements ou leur manque au niveau de chaque patient. C'est dire que les instruments que nous construisons sont généraux et qu'ils ne mesurent pas les difficultés spécifiques de plusieurs sousgroupes de patients, tels par exemple ceux qui ont des problèmes sexuels, ceux qui sont arriérés ou ceux qui se mutilent, etc. Il y a par ailleurs un groupe de criminels avec lesquels on n'a pas réussi la première étape. Il s'agit d'hommes qui ont purgé une longue sentence aux pénitenciers et qui demeurent à l'IPPM pour terminer leurs deux dernières années. Ils sont d'un certain âge et semblent avoir terminé leur carrière criminelle; cependant, ils ne connaissent pas d'autres styles de vie. En somme, les projets de recherche ne manquent pas!

\section{L'étude de post-cure}

L'étude de "post-cure " des ex-patients constitue le deuxième volet du projet d'évaluation. Les buts de cette étude sont de différents ordres et partiellement déterminés par les contraintes budgétaires. Comme il n'y a pas d'étude de "follow-up 》 des ex-patients, on ne connaît pas leur taux de récidive criminelle et de rechute psychiatrique. Et la littérature scientifique en ce domaine ne nous aide pas à combler cette lacune; elle est tout aussi inexistante (Quinsey $\&$ Boyd, 1977). On a donc affaire à un problème sérieux. S'il nous est difficile de connaître les cas de réussite car les ex-patients ne reviennent pas sur les lieux pour nous dire qu'ils vont bien, il en va tout autrement des cas qui ont échoué. Ces derniers sont soit réadmis en institution ou font parler d'eux dans les journaux à la suite de crimes à sensation. Ces informations non systématiques ont pour effet de décourager le personnel traitant sans fournir les éléments nécessaires pour améliorer les programmes de traitement. Même si une telle étude s'impose, il n'est pas possible de la réaliser pour l'instant car elle serait trop coûteuse.

Voici les quatres objectifs du projet de post-cure :

1. rester en contact avec les ex-patients de façon à pouvoir les retracer rapidement le jour où nous pourrons entreprendre une étude de plus grande envergure;

2. établir les taux de récidive criminelle et de rechute psychiatrique par type de patient;

3. essayer d'établir les profils d'individus qui réussissent et de ceux qui ne réussissent pas pour chaque programme de traitement;

4. valider nos instruments d'évolution. 
Afin de remplir ces objectifs, nous avons retenu la méthodologie suivante. Tous les six mois, nous contacterons tous les ex-patients et nous les inviterons à répondre par téléphone à un court questionnaire d'une durée de cinq ou six minutes. On pourra ainsi recueillir des informations concernant leur lieu d'habitation, soit un hôpital, une prison, un foyer de groupe, un appartement, etc., leurs sources de revenu, leur emploi, leurs contacts avec des professionnels des services publics et leurs contacts sociaux. Tous les six mois également, on élargira l'échantillonnage. Du ler au 15 mars de cette année, on commencera la première cueillette d'informations.

Ces deux volets de la recherche évaluative - les instruments à mesurer l'évolution et l'étude de post-cure - nous fourniront la matière de base pour améliorer les comportements d'interventions ainsi que pour faire de la recherche relative aux processus de traitement. De tels projets seront réalisés avec des subventions venant de l'extérieur ou encore dans le cadre d'un mémoire de maîtrise ou d'une thèse de doctorat. Il s'agira donc pour chaque unité de soins d'examiner leur programme de traitement à partir des divers éléments du processus d'interaction avec le client et d'en étudier minutieusement les résultats. Ces informations nous permettront de raffiner notre intervention et de mieux orienter la partie théorique du programme.

\section{L'étude d'isolement}

Le projet d'isolement contient plusieurs buts. Dans le contexte de l'IPPM où le personnel en général ne voyait pas l'utilité de cette recherche, il était important de le convaincre non seulement de sa nécessité absolue mais aussi de celle de le former pour y participer.

1.Le premier objectif du projet d'isolement est de former des équipes en vue de recueillir certaines informations de façon plus systématique et complète et de distribuer à l'intérieur de chaque équipe des tâches bien précises de manière à respecter un échéancier pré-déterminé.

2.Le deuxième objectif consiste à démontrer aux équipes de cliniciens que le Service de Recherche peut travailler à partir de leurs informations de façon à produire des analyses pertinentes à leur travail.

3.Le troisième objectif de cette étude est de former le personnel clinique à l'utilisation de ces rapports en vue d'améliorer leurs interventions.

4.Le quatrième objectif est d'un tout autre ordre : il s'agit d'établir les différents types et le niveau de violence à l'intérieur de l'institution ainsi que d'évaluer les différentes formes d'i- 
solement de l'IPPM. Plus précisément, il s'agirait d'établir le but thérapeutique de chaque forme d'isolement. Par exemple, l'isolement pourrait être utilisé pour punir un patient qui a commis un acte interdit, ou pour l'empêcher de commettre un acte violent, ou encore pour le faire calmer, ou le faire réfléchir sur un point spécifique.

C'est en vue de déterminer dans quelle situation et avec quel type de patient chaque forme d'isolement atteint un but thérapeutique qu'il faut accomplir cette étude.

Suite aux discussions que nous avons tenues avec le personnel clinique et administratif et suite aux nombreuses révisions de différents instruments, nous sommes parvenus à mettre au point une fiche qui sera remplie par le membre du personnel qui mettra un patient en isolement. Cette fiche indiquera le nom du patient, le type d'isolement, la raison pour laquelle le patient est isolé, la durée et le nom de l'individu qui a pris la décision. Ces données seront compilées à la fin de chaque mois et envoyées au Service de Recherche qui en fera une analyse sommaire au niveau de chaque unité et qui en publiera un rapport. À la fin de chaque année, une analyse plus poussée répondra aux questions pertinentes du personnel clinique de chaque unité, aux questions concernant la politique d'isolement de l'hôpital et aux questions émergeant de la littérature scientifique. Entre-temps, des professionnels de chaque unité seront formés de façon à ce qu'ils puissent utiliser ces informations en vue d'améliorer le fonctionnement de leurs équipes et l'efficacité de leurs interventions.

\section{Conclusion}

Comme nous l'avons constaté, l'Institut Philippe Pinel de Montréal est à la fois un hôpital psychiatrique et une prison. On y trouve diverses catégories de personnes, mais toujours des individus qui ont des difficultés psychologiques graves. Leurs problèmes ainsi que les traitements dont ils bénéficient, ne les distinguent en rien des patients des autres hôpitaux psychiatriques. Il n'en reste pas moins qu'on y distingue les détenus qui ont accepté de participer aux programmes expérimentaux de réhabilitation : les criminels qui ont développé des difficultés psychologiques pendant une période d'incarcération, et les personnes ayant des déficiences intellectuelles.

Sommairement, le mandat de l'IPPM consiste à aider ce type de personnes à reprendre une vie sociale active à l'extérieur des ins- 
titutions. L'institut doit en même temps veiller à ce que le retour de ces patients dans un milieu normal ne constitue pas une menace pour la société.

Mais, comment peut-on atteindre un tel objectif ? Ni la littérature scientifique, ni la pratique courante ne nous fournissent à cet égard une réponse adéquate.

Un tel vide de connaissances nous laisse sans aucun mode d'interventions efficaces auprès de ces différents types de clientèle. Nous devrons alors adopter une stratégie d'évaluation qui vise à intégrer le travail clinique et la recherche. Dans cette optique, nous procéderons donc à dégager les éléments positifs et négatifs des programmes de traitement afin de les raffiner et de les rendre plus efficaces. Pour atteindre cet objectif, il faut évaluer les changements chez les patients de façon continue pendant leur séjour à l'IPPM ainsi que par la suite.

Le projet d'évaluation qui est en cours présentement a trois volets : le développement des instruments qui mesureront l'évolution des patients; l'étude post-cure des ex-patients; et une étude de l'utilisé des différentes formes d'isolement. L'ensemble de cette recherche fournira les données essentielles pour le développement de programmes d'interventions dans ce domaine. Il ne reste qu'à espérer que ceux qui détiendront le pouvoir à ce moment-là, vont s'en servir.

\section{BIBLIOGRAPHIE}

BERGIN, A.E. \& GARFIELD, S.L. (1978) : Handbook of Psychotherapy and Behavior Change, New York, Wiley.

HODGINS, Sheilagh : "L'évaluation des programmes de réhabilitation : comment la faire ", Revue Internationale de Criminologie et Police Technique, sous presse.

LAMBERT, M.J., BERGIN, A.E. \& COLLINS, J.L. (1977) : «Therapist induced deterioration in psychotherapy ", dans A.S. Gurman et A.M. Razin (Eds); Effective Psychotherapy, A Handbook of Research, New York, Pergamon Press, 452-481.

QUINSEY, Vernon L. (1981) : "The long-term management of the mentally abnormal offender, dans S.J. Hucker, C.D. Webster et M.H. Ben-Aron (Eds.) : Mental Disorder and Criminal Responsability, Toronto, Butterworths.

QUINSEY, V.L. \& BOYD, B.A. (1977) : "An assessment of the characteristics and dangerousness of patients held on warrants of the Lieutenant-Governor ", Crime and Social Justice, 268-274.

RACHMAN, S.J. \& WILSON, G.T. (1980) : The Effects of Psychological Therapy, Toronto, Pergamon Press.

SEECHREST, Lee, WHITE, Susan O \& BROWN, Elizabeth D. (1979) : The Rehabilitation of Criminal Offenders : Problems and Prospects, Washington (D.C.), National Academy of Sciences. 